\title{
Artificial Intelligence and Relativistic Computing
}

\author{
Y.N. Zayko* \\ Russian Presidential Academy of National Economy and Public Administration, Stolypin Volga Region Institute, \\ Saratov, Russia.
}

*Corresponding Author: Y.N. Zayko, Russian Presidential Academy of National Economy and Public Administration, Stolypin Volga Region Institute, Saratov, Russia.

\begin{abstract}
The article considers the fundamental question of the non-computable nature of algorithms that could serve as a basis for modeling brain consciousness and, therefore, for the develop-ment of artificial intelligence systems. The use of relativistic algorithms to overcome the Turing barrier is proposed. Quantum and relativistic algorithms are compared. The advantage of relativistic algorithms is shown
\end{abstract}

Keywords: artificial intelligence, non-computable algorithm, relativistic computing, Turing barrier

\section{INTRODUCTION}

The main task facing the developers of artificial intelligence systems (AI) is to unravel working and simulate the human brain, or, more specifically, to clarify the nature of its activities associated with awareness. All other aspects of the activity in the field of AI, one way or another, are associated with the solution of technical problems aimed at the construction of "smart" robots.

Currently, the prevailing point of view on the nature of intelligence, expressed by R. Penrose [1], according to which one can model the work of the brain associated with consciousness only with the use of non-computable (in the sense of A. Turing) algorithms. R. Penrose also proposes to consider the processes occurring at the cellular level and admitting a substantially quantum description as a physical realization of the consciousness function of the living brain.

In the report, as a basis for modeling consciousness, the so-called relativistic algorithms are considered i.e. algorithms specific to relativistic computing.

What is relativistic computing?

The term "relativistic computer" (RC) first appeared in the works of I. Nemeti (with co-authors) in 1987 [2, 3]. It was supposed to use relativistic effects to overcome the limitations imposed on the calculations carried out in the framework of the model of the classical Turing machine (MT) - "Turing barrier". In particular, it was suggested that the RC can be used to solve the so-called non-computable tasks that cannot be solved on MT (requires infinite time). For this, it was proposed to use the achievements of relativistic physics - Kerr-Newman black holes, the space-time metric near which has singularities that ensure the natural adopting of infinity in the number of allowed computation time values. However, since the implementation of such projects to this day remains problematic, they have no practical consequences. In the works of the author [4 - 7], the idea of the RC received a practical embodiment in the form of developing an algorithm for calculating the sums of divergent series - a task also related to the problems which are non-computable on MT.

The structure of the report is as follows. The next section (2) is devoted to a brief presentation of the arguments of R. Penrose in favor of approach $\boldsymbol{C}$. Section 3 discusses the use of relativistic computing technology as the basis for realizing the point of view $\boldsymbol{C}$. In the Discussion section (4), we compare the arguments of R. Penrose and those proposed in the report. The conclusion contains the main results of the report.

\section{R. PENROSE'S APPROACH.}

R. Penrose identifies four main points of view on the nature of conscious thinking [1]

"A. All thinking is computation; in particular, feelings of conscious awareness are evoked merely by the carrying out of appropriate computations. 
B. Awareness is a feature of the brain's physical action; and whereas any physical action can be simulated computationally, computational simulation cannot be itself evoke awareness.

$\boldsymbol{C}$. Appropriate physical action of the brain evokes awareness, but this physical action cannot even be properly simulated computationally.

D. Awareness cannot be explained by physical, computational, or any other scientific terms. "”

Analyzing these points of view, R. Penrose makes convincing arguments in favor of $\boldsymbol{C}$. He writes "According to $\boldsymbol{C}$, the problem of conscious awareness is indeed a scientific one, even if the appropriate science may not yet be at hand"[1].

Moreover, he is trying to find physical processes in living organisms that could claim to be the "generator" of consciousness, emphasizing their essentially uncalculable nature ${ }^{2}$. As such, he indicates the quantum behavior of some components of living organisms, which should provide two essential points:

1. A high degree of quantum coherence of the unitary evolution of a quantum information carrier over a period of time sufficient to accumulate qualitative characteristics that would allow talking about its "conscious" status.

2. The presence of a special procedure $\left(\boldsymbol{R}\right.$ or $\left.\boldsymbol{O} \boldsymbol{R}^{3}\right)$, which has a substantially non-computable nature, the essence of which is the destruction of the quantum-coherent state of the accumulated information during measurement, the role of which plays the awareness of this information.

The hypothetical procedure $\boldsymbol{R}$ often discussed in the literature devoted to the fundamental issues of quantum theory, or rather $\boldsymbol{O} \boldsymbol{R}$ performs the "contraction" of the wave function, which represents the quantum superposition of individual states, providing the observed macroscopic value of the quantity which is written in the quantum packet.

\section{RELATIVISTIC COMPUTING AS AN AI SOURCE.}

Relativistic calculations can serve as an alternative source of AI. Infinite counting time for them is not an indication of unsuitability for work. Moreover, since relativistic calculations are characterized by the presence of singularities of the manifold on which the calculations are performed, the infinite runtime is associated with their Turing non-computability. Recall that the manifold on which computable algorithms are executed is the infinite Euclidean number line.

So, in [4], it was calculated the sum of the divergent series for the Riemann zeta function $\zeta(-1)$

$$
\varsigma(-1)=\sum_{n=1}^{\infty} n \approx-0,08035
$$

which is matching with exact value $-B_{2} / 2=-1 / 12$ [8] with relative error $\sim 3,576 \%\left(B_{n}-\right.$ Bernoulli numbers, $n=2$ ). The calculation is based on the fact that the partial sums of series (1) coincide with the formula for the distance traveled by a material point moving along a straight line with constant acceleration. From a physical point of view, these conditions are realized in the gravitational

\footnotetext{
${ }^{1}$ In short, these points of view mean that consciousness allows $(\boldsymbol{A})$ (does not allow $(\boldsymbol{B})$ ) mathematical modeling; admits, but using non-computable algorithms $(\boldsymbol{C})$; unknowable $(\boldsymbol{D})$

2 In the original, the author uses the terms "non-computational" and "non-computable" as synonyms, specifically stipulating that we are talking about Turing computability ([1], p. 394). In Russian, these terms have different meanings, what is mentioned below.
}

${ }^{3}$ Abbreviation of Reduction or Objective Reduction [1] 
field of the massive infinite plane $Y O Z$ perpendicular to the line of motion of the point $O X$. The metric on the line $O X$ is found from Einstein's equations and has the form

$$
\begin{aligned}
& d s^{2}=\left(1+\frac{x}{x_{c}}\right) c^{2} d t^{2}-\left(1+\frac{x}{x_{c}}\right)^{-1} d x^{2}-d y^{2}-d z^{2} \\
& x_{c}=\frac{4 \pi \sigma K}{c^{2}}
\end{aligned}
$$

where $K$-gravitational constant, $\sigma$ - mass density of the plane $Y O Z$. To calculate the sum of series (1), the relativistic equation of motion was solved with the initial data obtained from the form of partial sums of series (1).

The calculation of the sum (1) in the form of a finite expression is possible in the metric (2), while in the flat metric the problem of determining the sum of the

series (1) relates to non-computable Turing problems ${ }^{4}$. This consideration allows us to talk about the relativity of the concept of computability - a problem that seemed to be non-computable in a flat metric becomes computable in a curved metric.

\section{DISCUSSION}

Thus, comparing the approach of R. Penrose with the approach using relativistic calculations proposed in this paper, we can draw the following conclusions:

1. The approach of R. Penrose is based on the poorly studied quantum properties of matter, while relativistic calculations are mainly associated with classical properties.

2. R. Penrose's approach is based on some hypotheses that have not been experimentally confirmed, while relativistic calculations are easily implemented in practice.

In particular, series (1) is found in the well-known Casimir problem of calculating the interaction force between two charged planes [9].

The advantage of relativistic computing as a candidate for the role of the carrier of AI over quantum computing is its feasibility. Indeed, while the properties of quantum computing are under discussion, especially concerning the properties of the $\boldsymbol{O} \boldsymbol{R}$ procedure, relativistic calculations have already been successfully used, for example, in calculations with divergent series [4]. Relativistic calculations have also worked well when considering problems in field theory containing divergences [10].

\section{CONCLUSION}

The article considers the fundamental question of the non-computable nature of algorithms that could serve as a basis for modeling brain consciousness and, therefore, form the basis for the development of artificial intelligence systems. A comparison of quantum and relativistic calculations is carried out. The advantage of relativistic calculations is shown.

\section{REFERENCES}

[1] Penrose, R., Shadows of the Mind. A Search for the Missing Science of Consciousness. Oxford Univ. Press, NY, Oxford, 1989.

[2] Nemeti I., David G. Relativistic Computers and the Turing Barrier// Applied Mathematics and Computation - 2006.-178, - P. 118-142.

[3] Andréka H., Németi I., Németi P. General relativistic hypercomputing and foundation of mathematics// Natural Computing.- 2009.- V. 8- № 3.- pp 499-516.

[4] Zayko Y.N. The Geometric Interpretation of Some Mathematical Expressions Containing the Riemann

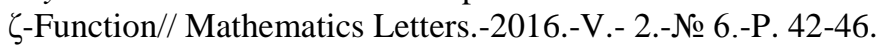

[5] Zayko Y. N. Calculation of the Riemann Zeta-function on a Relativistic Computer// Mathematics and Computer Science.-2017.- V. 2.- № 2.-P. 20-26.

${ }^{4}$ To clarify the terminology, we emphasize that the expression of the sum of the series (1) in terms of Bernoulli numbers is obtained by applying the Euler-Maclaurin formula and is a non-computational procedure. 
[6] Zayko Yu. N. The Proof of the Riemann Hypothesis on a Relativistic Turing Machine// International Journal of Theoretical and Applied Mathematics.- 2017.- V. 3.-№ 6.- P. 219-224,

[7] Zayko Yu. N. The Second Postulate of Euclid and the Hyperbolic Geometry// International Journal of Scientific and Innovative Mathematical Research.- 2018.- V. 6.- № 4.- P 16-20; arXiv: 1706.08378, 1706.08378v1 [math.GM])

[8] E. Janke, F. Emde, F. Lösch, Tafeln Höherer Funktionen, Stuttgart: B.G. Teubner Verlagsgeselschaft (1960).

[9] H. B. G. Casimir, On the attraction between two perfectly conducting plates // Proceedings of the Koninklijke Nederlandse Akademie van Wetenschappen: journal. 1948. Vol. 51. P. 793-795.

[10] Zayko Y.N. Quantum Field Theory Free of Divergences, Global Journal of Science Frontier Research A: Physics and Space Science, V. 20, I. 1, 2020, pp. $27-31$.

Citation: Y.N. Zayko Artificial Intelligence and Relativistic Computing, International Journal of Scientific and Innovative Mathematical Research (IJSIMR), vol. 8, no. 5, pp. 21-24, 2020. Available : DOI: https://doi. org/10.20431/2347-3142.0805004

Copyright: (C) 2020 Authors. This is an open-access article distributed under the terms of the Creative Commons Attribution License, which permits unrestricted use, distribution, and reproduction in any medium, provided the original author and source are credited. 